\title{
Temperamento de crianças em idade pré-escolar
}

\section{Temperament of preschool children}

\section{Beatriz Schmidt*}

Universidade Federal de Santa Catarina - UFSC, Florianópolis, Santa Catarina, Brasil.

\section{Carina Nunes Bossardi**}

Universidade Federal de Santa Catarina - UFSC, Florianópolis, Santa Catarina, Brasil.

\section{Lauren Beltrão Gomes***}

Universidade Federal de Santa Catarina - UFSC, Florianópolis, Santa Catarina, Brasil.

\section{Maria Aparecida Crepaldi**}

Universidade Federal de Santa Catarina - UFSC, Florianópolis, Santa Catarina, Brasil.

Mauro Luis Vieira*****

Universidade Federal de Santa Catarina - UFSC, Florianópolis, Santa Catarina, Brasil.

\section{I ntrodução}

Associam-se ao risco de desenvolvimento de psicopatologias algumas características da pessoa, como o temperamento, que consiste em uma importante variável individual que interage com variáveis ambientais e influencia trajetórias desenvolvimentais de crianças, relacionando-se a desfechos adaptativos ou desadaptativos (KLEIN; LINHARES, 2010). O temperamento é compreendido como diferenças individuais com base constitucional na reatividade e na autorregulação, observadas nos domínios de emocionalidade, atividade motora e atenção, sendo influenciadas ao longo do tempo pela hereditariedade, maturação e experiência. A reatividade é definida como característica de responsividade individual a mudanças de estimulação e por meio de parâmetros de latência, tempo de aumento, intensidade máxima e tempo de recuperação da reação. A autorregulação consiste em processos que modulam a reatividade, incluindo aproximação/retraimento comportamental, controle inibitório e de atenção (ROTHBART, 2004; KLEIN; LINHARES, 2010). O temperamento é composto por três fatores: (1) extroversão, caracterizado pelas dimensões impulsividade, prazer de alta 
intensidade, nível de atividade e timidez; (2) afeto negativo, definido pelas dimensões tristeza, medo, raiva, desconforto e capacidade de se acalmar; (3) controle com esforço, que diz respeito às dimensões controle inibitório, focalização de atenção, prazer de baixa intensidade e sensibilidade perceptual (ZENTNER; BATES, 2008).

$O$ afeto negativo e a extroversão estão associados à reatividade e aparecem desde as fases mais primevas do desenvolvimento, podendo ser observados com clareza já nos primeiros meses de idade da criança. O controle com esforço, em contrapartida, associa-se à autorregulação e começa a se delinear no final do primeiro ano, tornando-se progressivamente mais sofisticado e organizado à medida que a criança se aproxima da fase pré-escolar (HILLSODERLUND; BRAUNGART-RIEKER, 2008). Sendo assim, apesar de apresentar uma relativa estabilidade temporal, o temperamento se desenvolve ao longo da trajetória de vida (ELSE-QUEST; HYDE; GOLDSMITH; VAN HULLE, 2006).

Estudos indicam que as características do temperamento estão associadas a desfechos desenvolvimentais nas crianças. Gunnar, Sebanc, Tout, Donzella e Van Dulmen (2003) identificaram que altos níveis de extroversão e o baixo controle com esforço estão associados positivamente à agressividade e, por consequência, à rejeição por pares em idade pré-escolar. Segundo Rothbart (2007), o baixo controle com esforço está associado ainda a problemas de externalização, enquanto o medo e a timidez se associam a problemas de internalização. As disposições temperamentais evocam reações em outras pessoas, o que faz aumentar ou diminuir o risco para psicopatologia; ademais, elas influenciam ainda a forma, o curso e a recorrência de desordens psicológicas (ROTHBART, 2004).

Considerando-se a relevância das diferenças individuais na trajetória de desenvolvimento infantil, o presente trabalho teve como objetivo caracterizar o temperamento de crianças com idade entre quatro e seis anos. Este estudo se insere no âmbito de um projeto de pesquisa mais abrangente, intitulado "A transmissão intergeracional da violência: a relação do conflito conjugal e parental com a agressividade entre pares de crianças de quatro a seis anos". Tal projeto vem sendo desenvolvido, desde o ano de 2009, por pesquisadores brasileiros vinculados à Universidade Federal de Santa Catarina, em parceria com o Canadá, mais especificamente a Universidade de Montreal e a Universidade do Québec em Montreal.

\section{Método}

\subsection{Participantes}


A amostra, constituída por conveniência, foi composta por 104 famílias biparentais. As 104 crianças focais (47 do sexo feminino e 57 do sexo masculino) tinham idade entre quatro e seis anos, frequentando a pré-escola no momento da coleta de dados. Ressaltase que tais crianças não haviam sido previamente identificadas por apresentar problemas de desenvolvimento ou potencial de risco para tanto (amostra populacional), já que o estudo intencionou compreender $o$ temperamento em relação aos processos desenvolvimentais normativos ou típicos.

\subsection{I nstrumento}

O temperamento infantil foi avaliado com base nas respostas de pai e de mãe à very short form do Children's Behavior Questionnaire (CBQ). Tal instrumento, desenvolvido por Putnam e Rothbart (2006), contempla questões referentes às reações infantis em diferentes situações. Trata-se de uma escala de sete pontos, que varia de 1 (totalmente falsa) a 7 (totalmente verdadeira), além do item "não se aplica". O CBQ é composto por três sub-escalas: extroversão (12 itens; a 0,75$)$, afeto negativo (12 itens; a 0,72 ) e controle com esforço (12 itens; a 0,74).

\subsection{Procedimentos de coleta de dados}

Foram enviadas cartas-convite às famílias, por intermédio de 23 instituições de educação infantil localizadas em um Estado da região sul do Brasil. A coleta de dados ocorreu nas residências dos participantes.

\subsection{Procedimentos éticos}

Os participantes assinaram o Termo de Consentimento Livre e Esclarecido. A pesquisa foi aprovada pelo Comitê de Ética em Pesquisa com Seres Humanos da Universidade Federal de Santa Catarina (Certificado no 520/2009).

\subsection{Procedimentos de análise dos dados}

Os dados obtidos foram tabulados e submetidos a análises estatísticas mediante a utilização do Statistical Package for Social Sciences (SPSS) - versão 18.0. Adotou-se tratamento estatístico não paramétrico, vez que nem todas as variáveis obedeceram à distribuição normal, o que foi identificado com a aplicação do teste Kolmogorov-Smirnov. 


\section{Resultados e discussão}

No fator extroversão, a média obtida com as respostas das mães ao $\mathrm{CBQ}^{1}$ foi $4,74(\mathrm{DP}= \pm 0,95)$ e dos pais foi $4,82(\mathrm{DP}= \pm 0,73)$. Sendo assim, mãe e pai apontaram, em média, como nem verdadeira nem falsa (4), ou como razoavelmente verdadeira (5) para o seu filho, situações tais quais: parece estar sempre com muita pressa para ir de um lugar ao outro (nível de atividade); gosta de descer em escorregadores altos ou de outras atividades de aventura (prazer de alta intensidade); parece estar à vontade com quase todas as pessoas (timidez); frequentemente entra rapidamente em novas situações (impulsividade).

No que diz respeito ao afeto negativo, as médias das respostas maternas $(M=4,98 ; D P= \pm 0,91)$ e paternas $(M=4,98 ; D P= \pm 0,91)$ indicam como nem verdadeiras nem falsas (4), ou como razoavelmente verdadeiras (5), as seguintes reações da criança: fica muito frustrada quando não lhe deixam fazer algo que quer (raiva); fica muito incomodada com um machucado ou corte pequeno (desconforto); tende a ficar triste se os planos da família não dão certo (tristeza); tem medo de ladrões ou do "bicho-papão" (medo); quando está zangada com alguma coisa, tende a ficar aborrecida por dez minutos ou mais (capacidade de se acalmar).

O fator que recebeu os maiores escores médios nas crianças focais foi o controle com esforço. Desse modo, mães $(M=5,74 ; D P= \pm 0,67)$ e pais $(M=5,63 ; \quad D P= \pm 0,63)$ consideram, em média, como razoavelmente verdadeiro (5) e bastante verdadeiro (6) que sua criança fica muito concentrada quando está desenhando ou pintando em um livro (focalização de atenção), que ela segue instruções (controle inibitório), que gosta de atividades rítmicas suaves, como se balançar (prazer de baixa intensidade) e que comenta quando um dos pais muda a aparência (sensibilidade perceptual). Crianças com altos níveis de controle com esforço apresentam estratégias de enfrentamento mais adaptativas em situações ansiogênicas, demonstrando um sofisticado nível de regulação emocional (CRAWFORD; SCHROCK; WOODRUFF-BORDEN, 2011). Assim, altos escores nesse fator estão relacionados a melhores desfechos desenvolvimentais nos âmbitos afetivo e social (KLEIN; LINHARES, 2010). Considera-se, portanto, que os altos escores médios (>5) em controle com esforço no grupo de crianças avaliado se constituem em um fator de proteção ao desenvolvimento infantil.

$O$ teste Mann-Whitney foi aplicado às médias maternas e paternas obtidas nos três fatores do $C B Q$, revelando que não há diferença estatisticamente significativa entre as respostas dos cônjuges, o que indica que eles concordam com relação às características de extroversão, afeto negativo e controle com esforço da sua criança. Tais achados são congruentes com Putnam e Rothbart (2006), uma 
vez que esses autores, no artigo que aborda o processo de desenvolvimento da very short form do CBQ, apontam para o bom grau de concordância entre mãe e pai no que concerne ao temperamento da criança focal.

Com o objetivo de investigar diferenças nos fatores do temperamento em virtude das distintas idades, utilizou-se o teste Kruskal-Wallis. As crianças foram divididas em três grupos de análise por faixa etária (quatro, cinco e seis anos). Foram constatadas diferenças nas respostas das mães sobre controle com esforço $\left(X^{2}=10,99 ; p<0,01\right)$. As médias obtidas por intermédio das respostas maternas ao fator controle com esforço foram progressivamente maiores, de acordo com a idade das crianças. Assim, a média do referido fator foi 5,51 $(D P=0,65)$ nas crianças com quatro anos; $5,86 \quad(D P=0,61)$ nas crianças com cinco anos; e 6,13 ( $D P=0,76)$ nas crianças com seis anos.

Esse resultado é compatível com dados da literatura, uma vez que, embora o temperamento seja relativamente estável ao longo do tempo, o processo de desenvolvimento influencia o modo como ele se apresenta (ELSE-QUEST et al., 2006). Nesse sentido, é natural que o controle com esforço seja maior em crianças de seis anos em comparação às crianças com menor idade, vez que tal fator tende a ficar mais sofisticado e organizado com o passar do tempo (HILLSODERLUND; BRAUNGART-RIEKER, 2008). Apesar da origem constitucional do controle com esforço, o desenvolvimento desse fator faz parte do processo de socialização, durante o qual as crianças podem aprender a controlar seus impulsos comportamentais e emocionais, apresentando reações e comportamentos mais aceitos socialmente (KARREMAN; VAN TUIJL; VAN AKEN; DEKOVIC, 2008).

\section{Considerações finais}

Em síntese, identificou-se que o controle com esforço recebeu os maiores escores médios no grupo avaliado, constituindo-se, portanto, em um fator de proteção ao desenvolvimento das crianças focais. Os fatores extroversão e afeto negativo se caracterizaram como nem verdadeiros nem falsos, ou razoavelmente verdadeiros, de acordo com as respostas maternas e paternas. Não foram identificadas diferenças significativas entre as médias dos três fatores respondidos pelas mulheres e pelos homens, sugerindo que ambos concordam quanto ao temperamento dos filhos. Outrossim, identificou-se ainda que as médias de controle com esforço foram progressivamente maiores de acordo com a idade das crianças focais, o que é compatível com achados da literatura.

Enfatiza-se que, por intermédio da identificação das disposições temperamentais infantis, o profissional de saúde mental pode intervir 
preventivamente, assumindo um papel de orientação aos cuidadores, auxiliando-os no processo de compreensão das diferenças individuais e dando suporte para que aprendam como manejá-las. Espera-se, com base nessas orientações, um aumento da responsividade e da sensibilidade dos cuidadores no processo de interação com as crianças, o que atua como fator de proteção à saúde mental infantil (KLEIN, 2009). Nesse sentido, destaca-se a importância de se considerar as diferenças individuais do temperamento na prática profissional, haja vista que pesquisas sobre temperamento e psicopatologia, embora recentes, revelam correlação positiva entre os dois fenômenos (PUTNAM; ROTHBART, 2006).

\section{Referências}

CRAWFORD, N. A.; SCHROCK, M.; WOODRUFF-BORDEN, J. Child internalizing symptoms: contributions of child temperament, maternal negative affect, and family functioning. Child Psychiatry Human Development, Nova Iorque, v. 42, p. 53-64, 2011.

ELSE-QUEST, N. M.; HYDE, J. S.; GOLDSMITH, H. H.; VAN HULLE, C. A. Gender differences in temperament: a meta-analysis. Psychological Bulletin, Berkeley, v. 132, n. 1, p. 33-72, 2006. GUNNAR, M. R.; SEBANC, A. M.; TOUT, K.; DONZELLA, B.; VAN DULMEN, M. M. H. Peer rejection, temperament and cortisol activity in preschoolers. Developmental Psychobiology, Malden, v. 43, n. 4, p. 346-358, 2003.

HILL-SODERLUND, A. L.; BRAUNGART-REIKER, J. M. Early individual differences in temperamental reactivity and regulation: implications for effortful control in early childhood. Infant Behavior \& Development, Amsterdã, v. 31, p. 386-397, 2008.

KARREMAN, A.; VAN TUIJL, C.; VAN AKEN, M. A. G.; DEKOVIC, M. Parenting, coparenting, and effortful control in preschoolers. J ournal of Family Psychology, Washington, v. 22, n. 1, p. 30-40, 2008.

KLEIN, V. C. Reatividade à dor, temperamento e comportamento na trajetória de desenvolvimento de neonatos pré-termo até a fase pré-escolar. 2009. 240 f. Tese (Doutorado em Saúde Mental) - Programa Programa de Pós-Graduação em Saúde Mental, Universidade de São Paulo, 2009.

KLEI N, V. C.; LINHARES, M. B. M. Temperamento e desenvolvimento da criança: revisão sistemática da literatura. Psicologia em Estudo, Maringá, v. 15, n. 4, p. 821-829, 2010.

PUTNAM, S. P.; ROTHBART, M. K. Development of short and very short forms of the children's behavior questionnaire. J ournal of Personality Assessment, Londres, v. 87, n. 1, p. 103-113, 2006. ROTHBART, M. K. Commentary: differentiated measures of temperament an multiple pathways to child desorders. Journal of 
Clinical Child and Adolescent Psychology, Londres, v. 33, n. 1, p. 82-87, 2004.

ROTHBART, M. K. Temperament, development, and personality. Current Directions in Psychological Science, Londres, v. 16, n. 4, p. 207-212, 2007.

ZENTNER, M.; BATES, J. Child temperament: an integrative review of concepts, research programs, and measures. European Journal of Developmental Science, Landsdale, v. 2, n. 1/2, p. 7-37, 2008.

\section{Endereço para correspondência \\ Beatriz Schmidt}

Laboratório de Psicologia da Saúde, Família e Comunidade (LABSFAC). Centro de Filosofia e Ciências Humanas, Departamento de Psicologia. Universidade Federal de Santa Catarina - Campus Universitário, Trindade. CEP 88040-970, Florianópolis, SC, Brasil.

Endereço eletrônico: psi.beatriz@gmail.com

\section{Carina Nunes Bossardi}

Núcleo de Estudos e Pesquisas em Desenvolvimento Infantil (NEPeDI). Centro de Filosofia e Ciências Humanas, Departamento de Psicologia. Universidade Federal de Santa Catarina - Campus Universitário, Trindade. CEP 88040-970, Florianópolis, SC, Brasil.

Endereço eletrônico: carinabossard@yahoo.com.br

\section{Lauren Beltrão Gomes}

Laboratório de Psicologia da Saúde, Família e Comunidade (LABSFAC). Centro de Filosofia e Ciências Humanas, Departamento de Psicologia. Universidade Federal de Santa Catarina - Campus Universitário, Trindade. CEP 88040-970, Florianópolis, SC, Brasil.

Endereço eletrônico: laurenbeltrao@yahoo.com.br

\section{Maria Aparecida Crepaldi}

Laboratório de Psicologia da Saúde, Família e Comunidade (LABSFAC). Centro de Filosofia e Ciências Humanas, Departamento de Psicologia. Universidade Federal de Santa Catarina - Campus Universitário, Trindade. CEP 88040-970, Florianópolis, SC, Brasil.

Endereço eletrônico: maria.crepaldi@gmail.com

\section{Mauro Luis Vieira}

Núcleo de Estudos e Pesquisas em Desenvolvimento Infantil (NEPeDI). Centro de Filosofia e Ciências Humanas, Departamento de Psicologia. Universidade Federal de Santa Catarina - Campus Universitário, Trindade. CEP 88040-970, Florianópolis, SC, Brasil.

Endereço eletrônico: maurolvieira@gmail.com

Recebido em: 16/05/2012

Aceito para publicação em: 05/07/2012

Acompanhamento do processo editorial: Adriana Benevides Soares

\section{Notas}

* Mestre pelo Programa de Pós-Graduação em Psicologia da Universidade Federal de Santa Catarina; Psicóloga; Especialista em Saúde da Família.

** Mestre pelo Programa de Pós-Graduação em Psicologia da Universidade Federal de Santa Catarina; Psicóloga; Especialista em Intervenções Psicossociais.

*** Mestre pelo Programa de Pós-Graduação em Psicologia da Universidade Federal de Santa Catarina; Psicóloga; Especialista em Saúde da Família. 
Beatriz Schmidt, Carina Nunes Bossardi, Lauren Beltrão Gomes,

Maria Aparecida Crepaldi, Mauro Luis Vieira

Temperamento de crianças em idade pré-escolar

**** Professora do Departamento de Psicologia e do Programa de Pós-Graduação em Psicologia da Universidade Federal de Santa Catarina; Psicóloga; Mestre em Psicologia Clínica; Doutora em Saúde Mental.

***** Professor do Departamento de Psicologia e do Programa de Pós-Graduação em Psicologia da Universidade Federal de Santa Catarina; Psicólogo; Mestre em Psicologia Experimental; Doutor em Psicologia Experimental.

${ }^{1}$ Os escores do CBQ variam de 1 a 7. 